DRAFT VERSION JUNE 19, 2018

Preprint typeset using $\mathrm{LT}_{\mathrm{E}} \mathrm{X}$ style emulateapj v. 6/22/04

\title{
FORMATION OF ULTRACOMPACT X-RAY BINARIES IN DENSE STAR CLUSTERS
}

\author{
N. IVAnova ${ }^{1}$, F. A. RASio ${ }^{1}$, J. C. Lombardi ${ }^{2}$, JR., K. L. Dooley ${ }^{2}$, And Z. F. ProulX ${ }^{2}$ \\ Draft version June 19, 2018
}

\begin{abstract}
Bright, ultracompact X-ray binaries observed in dense star clusters, such as Galactic globular clusters, must have formed relatively recently, since their lifetimes as persistent bright sources are short (e.g., $\sim 10^{8}$ yr above $10^{36} \mathrm{erg} \mathrm{s}^{-1}$ for a $1.4 M_{\odot}$ neutron star accreting from a degenerate helium companion with an initial mass of $\sim 0.2 M_{\odot}$ ). Therefore, we can use the present conditions in a cluster core to study possible dynamical formation processes for these sources. Here we show that direct physical collisions between neutron stars and red giants can provide a sufficient formation rate to explain the observed numbers of bright sources. These collisions produce tight, eccentric neutron star - white dwarf binaries that decay to contact by gravitational radiation on timescales $\sim 10^{6}-10^{10} \mathrm{yr}$, usually shorter and often much shorter than the cluster age.
\end{abstract}

Subject headings: binaries: close — galaxies: star clusters — globular clusters: general — hydrodynamics — stellar dynamics - X-rays: binaries

\section{INTRODUCTION}

Ultracompact X-ray binaries (UCXBs) are persistent, bright X-ray sources $\left(L_{x} \sim 10^{36}-10^{39} \mathrm{erg} \mathrm{s}^{-1}\right)$ containing a neutron star (NS) accreting from a low-mass, degenerate companion in a very tight orbit of period $P \lesssim 1 \mathrm{hr}$. UCXBs may well be dominant among the bright low-mass X-ray binaries (LMXBs) observed in old globular clusters (GCs), both Galactic (Deutsch et al. 2000; van der Sluvs et al. 2005) and extragalactic (Bildsten \& Delove 2004). It was recognized 30 years ago that the total numbers of LMXBs observed in GCs clearly indicate a dynamical origin, with formation rates exceeding those in field populations by several orders of magnitude (Clark 1975). Indeed, the stellar encounter rate in a cluster core is an excellent predictor for the presence of a bright LMXB (Jordán et al. 2004).

The growing importance of UCXBs is clear from the role they have played recently in a number of different contexts. They may dominate the bright end of the X-ray luminosity function in elliptical galaxies (Bildsten \& Delove 2004). They pose a number of challenges to, and may allow us to test our fundamental physics of, stellar structure for low-mass degenerate or quasi-degenerate objects (Delove \& Bildsten 2003). They may also connect in a fundamental way to NS recycling, as suggested by the fact that three out of six accretion-powered millisecond X-ray pulsars known in our Galaxy are UCXBs (Chakrabartv 2004; Grebenev et al. 2004). Finally, UCXBs may well be the progenitors of the many eclipsing binary radio pulsars with very low-mass companions observed in GCs (Rasio et al. 2000).

Several possible formation processes for UCXBs are possible. Exchange interactions between NSs and primordial binaries provide a natural way of forming possible progenitors of UCXBs (Rasio et al. 2000). This may well dominate the formation rate when integrated over the entire history of a cluster. However, it is unlikely to be significant for bright UCXBs observed today. This is because the progenitors must be intermediate-mass binaries, with the NS companion massive enough for the initial mass transfer (MT) to become dynamically unstable, leading to common-envelope

\footnotetext{
${ }^{1}$ Northwestern University, Dept of Physics and Astronomy, Evanston, IL 60208.

2 Dept of Physics and Astronomy, Vassar College, Poughkeepsie, NY 12604.
}

(CE) evolution and significant orbital decay. Instead, all main-sequence stars remaining today in a GC have masses low enough to lead to stable MT (and orbits that expand during MT, leading to LMXBs with wide periods and nondegenerate donors). Alternatively, some binaries with stable MT could evolve to ultra-short periods by magnetic braking (Pylyser \& Savoniie 1988; Podsiadlowski et al. 2002). However, producing UCXBs through this type of evolution requires very careful tuning of initial conditions and is therefore very unlikely to explain most sources (?).

Verbunt (1987) first proposed that a physical collision between a NS and a red giant (RG) could lead to UCXB formation. In his scenario, the collision was assumed to lead directly to a CE system in which the NS and RG core would quickly inspiral. However, RG-NS collisions that occur now in old GCs (where RGs have low masses, close to $m_{\mathrm{to}}$ ) do $n o t$ lead to CE evolution. Instead, the RG envelope is promptly disrupted, leaving behind an eccentric NS-WD binary, as shown by Rasio \& Shapiro (1991) using 3-D hydrodynamic calculation. Nevertheless, if the post-collision NS-WD binaries can decay through gravitational-wave emission all the way to contact, they can still become UCXBs (Davies et al. 1992).

\section{OUTCOME OF COLLISIONS}

Using the 3-D Smoothed Particle Hydrodynamics (SPH) code StarCrash ${ }^{3}$ we have computed about 40 representative collisions between various RG stars and a $1.4 M_{\odot} \mathrm{NS}$ (Lombardi et al. 2004). In our models, both the NS and the RG core are represented by point masses coupled to the gas by (softened) gravity only (Rasio \& Shapiro 1991). Our initial RG models were calculated using the stellar evolution code described in detail in Podsiadlowski et al. (2002), Ivanova et al. (2003), and Kalogera et al. (2004). The models include stars on the subgiant branch (with total mass $M=0.8 M_{\odot}$, core mass $m_{\mathrm{c}}=0.10 M_{\odot}$ and radius $R_{\mathrm{rg}}=1.6 R_{\odot}$ and with $M=0.9 M_{\odot}, m_{\mathrm{c}}=0.12 M_{\odot}$ and $\left.R_{\mathrm{rg}}=2 R_{\odot}\right)$, and several models near the base of the RG branch. Our most evolved models have $M=0.9 M_{\odot}, m_{\mathrm{c}}=0.25 M_{\odot}$ and $R_{\mathrm{rg}}=6.8 R_{\odot}$. More evolved RGs contribute very little to the total collision rate (see $\S 3)$. The distance of closest approach for the initial collision varies from $r_{p}=0.1 R_{\mathrm{rg}}$ (nearly head-on) to

\footnotetext{
${ }^{3}$ See http://www.astro.northwestern.edu/StarCrash/.
} 


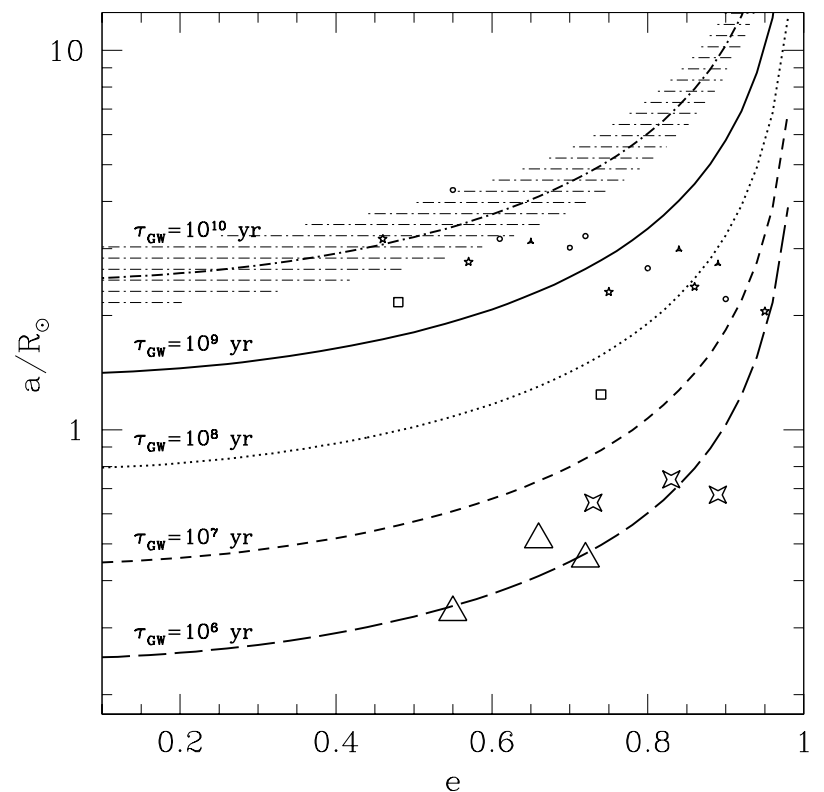

FIG. 1.- Dependence of the gravitational radiation merger time on postcollision semimajor axis $a$ and eccentricity $e$ for a binary consisting of a $1.4 M_{\odot}$ NS and a $0.25 M_{\odot}$ WD. The points with different symbols show the results of our SPH calculations for six different giant models (starred symbols are for a $0.9 M_{\odot}$ star; others are for a $0.8 M_{\odot}$ star at different evolutionary stages, e.g., triangles represent a subgiant, and circles our most evolved $\mathrm{RG}$ model). The symbol area is proportional to the collision rate, according to eq. (4), i.e., symbols for less evolved RGs appear larger. The hatched area shows how the merger time (here for the line of constant merger time $\tau_{\mathrm{gw}}=10^{10} \mathrm{yr}$ ) changes when we vary slightly the binary parameters: the upper boundary corresponds to a $1.5 M_{\odot} \mathrm{NS}$ with a $0.45 M_{\odot} \mathrm{WD}$, and the lower boundary corresponds to a $1.3 M_{\odot}$ NS with a $0.15 M_{\odot}$ WD.

$r_{p}=1.3 R_{\mathrm{rg}}$ (grazing).

In agreement with previous SPH calculations (Rasio \& Shapiro 1991; Davies et al. 1992), we find that all collisions produce bound systems in which the RG core ends up in a high-eccentricity orbit around the NS. However, in contrast to those older studies, our new SPH calculations extend over much longer times (up to $\sim 500$ successive pericenter passages), allowing us to determine accurately the final parameters of the orbit (Lombardi et al. 2004). Typically $\sim 50 \%$ of the $\mathrm{RG}$ envelope is ejected to infinity, while most of the rest becomes bound to the NS. Only about $\sim 0.1 M_{\odot}$ remains bound to the $\mathrm{RG}$ core, which will eventually cool to a degenerate WD (cf. §4). The material left bound to the NS will attempt to form an accretion disk as it cools. The fate of this material is rather uncertain. It could be accreted onto the NS and spin it up (in $\sim 10^{6} \mathrm{yr}$ at the Eddington limit), or, more likely, it could be ejected if the energy released by accretion couples well to the gas. With an efficiency $\epsilon$, the entire mass of gas could be ejected to infinity in as little as $\tau_{\text {gas }} \sim 500(\epsilon / 0.1)^{-1}$ yr. This very short lifetime justifies our assumption that the parameters of the post-collision orbits determined by our SPH calculations are nearly final, i.e., that the orbital parameters are no longer affected by coupling of the orbit to the residual gas.

When we apply the Peters (1964) equations to these postcollision systems, we find that most of them inspiral on rather short timescales (Fig. 1). Therefore, we assume for the rest of this paper that all RG-NS collisions can produce UCXBs.

\section{COLLISION RATE}

Consider a NS of mass $m_{\mathrm{ns}}$ in the core of a cluster containing $N_{*}$ ordinary stars (here we neglect binaries; see $\S 4$ ). If all these ordinary stars were turn-off stars of radius $R_{\text {to }}$ and mass $m_{\mathrm{to}}$, the collision rate for the NS would be

$$
\mathcal{R}_{\mathrm{to}} \equiv 2 \pi G\left(m_{\mathrm{to}}+m_{\mathrm{ns}}\right) N_{*} R_{\mathrm{to}} \sigma^{-1} V_{\mathrm{c}}^{-1},
$$

where $\sigma$ is the relative velocity dispersion, $V_{\mathrm{c}}$ is the core volume. Here we assume that the collision cross section is dominated by gravitational focusing .

To compute the collision rate with RGs, we take into account that the number of RGs, $d N_{\mathrm{rg}}$, within any small range of radii between $R_{\mathrm{rg}}$ and $R_{\mathrm{rg}}+d R_{\mathrm{rg}}$ is proportional to the time $d t$ spent there by the star as it ascends the RG branch, $d N_{\mathrm{rg}}=f_{\mathrm{rg}} N_{*} d t / \tau$. Here $f_{\mathrm{rg}}$ is the fraction of stars with masses close enough to the turn-off mass to have become RGs, and $\tau$ is the total lifetime (from the ZAMS to the end of the RG stage) of a turn-off star, only slightly larger than the cluster age. For a simple analytic estimate, we use the following approximate relation between age and radius (eq. A9 of Kalogera \& Webbink 1996),

$$
R_{\mathrm{rg}}(t) \simeq R_{\mathrm{ZAMS}}\left(1-\frac{t}{\tau}\right)^{-0.28}
$$

where we use $R_{\mathrm{ZAMS}} \simeq 0.7 R_{\mathrm{to}}$. Next we replace $d t$ by $d R_{\mathrm{rg}} /\left(d R_{\mathrm{rg}} / d t\right)=\left(R_{\mathrm{to}} / R_{\mathrm{rg}}\right)^{4.6} \tau d R_{\mathrm{rg}} / R_{\mathrm{to}}$. The collision rate for a NS with RGs between $R_{\mathrm{rg}}$ and $R_{\mathrm{rg}}+d R_{\mathrm{rg}}$ is

$$
d \mathcal{R}=2.6 \pi G\left(m_{\mathrm{to}}+m_{\mathrm{ns}}\right) R_{\mathrm{rg}} \sigma^{-1} V_{\mathrm{c}}^{-1} d N_{\mathrm{rg}} .
$$

Here a collision is defined to be any encounter with a distance of closest approach less than $1.3 R_{\mathrm{rg}}$, consistent with our $\mathrm{SPH}$ results. Integrating this over $R_{\mathrm{rg}}$ from the base of the RG branch, defined by setting $R_{\mathrm{rg}} \equiv b R_{\mathrm{to}}$, to the maximum radius of a $\mathrm{RG}, R_{\max } \gg b R_{\mathrm{to}}$, we find a total collision rate

$$
\mathcal{R}_{\mathrm{UCXB}} \simeq 0.51 f_{\mathrm{rg}} b^{-2.6} \mathcal{R}_{\mathrm{to}} .
$$

Alternatively, note that we could also directly integrate eq. (3) over time, without changing the variable from $t$ to $R_{\mathrm{rg}}$. Because the collision rate is linearly proportional to radius when gravitational focusing dominates, we can then write

$$
\mathcal{R}_{\mathrm{UCXB}} \simeq 2.6 \pi G\left(m_{\mathrm{to}}+m_{\mathrm{ns}}\right) N_{*} f_{\mathrm{rg}} \bar{R}_{\mathrm{rg}} \sigma^{-1} V_{\mathrm{c}}^{-1},
$$

where $\bar{R}_{\text {rg }}$ is the time-average radius of the RG. Using eq. (2) it is easy to show that eqs. (4) and (5) agree. Eq. (5) has the advantage that any stellar evolution treatment can be used to determine $\bar{R}_{\text {rg }}$, including fitting formulae more detailed than eq. (2) or numerical results from stellar evolution calculations.

The steep inverse dependence on $b$ in eq. (4) indicates that the collision rate is completely dominated by the smallest RGs: although the cross section increases (linearly) with radius, the faster stellar evolution at larger radii dominates, so that collisions are much more likely to happen when the star is just leaving the main sequence, i.e., on or close to the $s u b$ giant branch (Verbunt 1987). The corresponding core mass is also small, typically $m_{\mathrm{c}} \simeq 0.1 M_{\odot}$ for $m_{\mathrm{to}} \simeq 0.8 M_{\odot}$.

We now proceed to evaluate $f_{\mathrm{rg}}$. This depends on the mass function of stars in the cluster core, which we expect to be very different from the IMF because of mass segregation. Indeed, observations of cluster cores reveal flat or even slightly rising mass functions (e.g., Richer et al. 2004). Here we assume that the number of stars within $d m$ is proportional to $m^{\alpha} d m$, with $\alpha>-1$, between a minimum $m_{\min }$ and a maximum $m_{\mathrm{to}}+\Delta m$. The spread $\Delta m$ of masses along 
TABLE 1

UCXB FORMATION IN GALACTIC CLUSTERS

\begin{tabular}{llllllll}
\hline \hline \multicolumn{1}{c}{ Cluster } & $t_{\mathrm{rg}} / \tau$ & $\bar{R}_{\mathrm{rg}}$ & $\log \rho_{0}$ & $\sigma$ & $\log M_{\mathrm{t}}$ & $N_{100}$ & $N_{\text {min }}$ \\
\hline NGC 1851 & 0.071 & 6 & 5.7 & 10.4 & 6.0 & 0.11 & 0.85 \\
NGC 6624 & 0.087 & 4.2 & 5.6 & 5.4 & 5.2 & 0.14 & 0.18 \\
NGC 6652 & 0.076 & 5.4 & 4.8 & 5.9 & 5.4 & 0.02 & 0.05 \\
NGC 6712 & 0.070 & 5.9 & 3.0 & 4.3 & 5.0 & 0.0005 & 0.0004 \\
NGC 7078 & 0.034 & 7.1 & 6.2 & 12.0 & 6.1 & 0.16 & 1.62 \\
Terzan 5 & 0.10 & 4.3 & 6.1 & 10.6 & 5.6 & 0.27 & 0.87 \\
47 Tuc & 0.081 & 4.9 & 5.1 & 11.5 & 6.1 & 0.23 & 0.23 \\
\hline
\end{tabular}

Note. - The RG lifetime fraction $t_{\mathrm{rg}} / \tau$ and the average RG radius $\bar{R}_{\mathrm{rg}}\left(\right.$ in $R_{\odot}$ ) are calculated directly from our stellar evolution code and used in eq. 6 (with $\alpha=0$ ) and eq. 5. $\rho_{0}$ is the cluster core density (in $M_{\odot} \mathrm{pc}^{-3}$ ), $\sigma$ is the (1-D) velocity dispersion (in $\mathrm{km} \mathrm{s}^{-1}$ ) and $M_{\mathrm{t}}$ is the total cluster mass (in $M_{\odot}$ ). For Ter 5 and NGC 6652, $\log \rho_{0}$ is based on the luminosity density from Diorgovski 1993) and an adopted mass-to-light ratio of 2 . The value of $\log \rho_{0}$ for NGC 6652 appears rather uncertain (see, e.g., Pryor \& Mevlan 1993; Diorgovski 1993). Values of $\log M_{\mathrm{t}}$ for NGC 6652 and Ter 5 are from Gnedin et al. 2002); $\sigma$ for Ter 5 is from Gnedin et al. 2002) and for NGC 6652 from Webbink 1985). Otherwise $\log \rho_{0}, \sigma$ and $\log M_{\mathrm{t}}$ are from Pryor \& Mevlan 1993).

the RG branch is obtained from the mass-dependence of the main-sequence lifetime $t_{\mathrm{ms}}$. Adopting the simple scaling $t_{\mathrm{ms}}=\tau\left(m_{\mathrm{to}} / m\right)^{3.6}$ (Hurley et al. 2000) we get $\Delta t_{\mathrm{ms}}=$ $3.6 \tau\left(m_{\mathrm{to}} / m\right)^{3.6} \Delta m / m$. Setting $\Delta t_{\mathrm{ms}}=t_{\mathrm{rg}}$, the total time spent on the RG branch, and $m \simeq m_{\text {to }}$ gives $\Delta m \simeq 0.28 m_{\mathrm{to}} t_{\mathrm{rg}} / \tau$.

To be consistent with our previous definition of a RG having a radius $\geq b R_{\text {to }}$ and using again eq. (2) we derive $t_{\mathrm{rg}} / \tau=$ $0.3 b^{-3.6}$. We can now calculate $f_{\text {rg }}$ directly from IMF. Assuming $\Delta m \ll m_{\text {to }}$, and $m_{\text {min }} \ll m_{\text {to }}$ we get

$$
f_{\mathrm{rg}}=(\alpha+1) \frac{\Delta m}{m_{\mathrm{to}}} \simeq 0.28(\alpha+1) \frac{t_{\mathrm{rg}}}{\tau}=0.08(\alpha+1) b^{-3.6} .
$$

Combining this with eq (4) we obtain the result,

$$
\mathcal{R}_{\mathrm{UCXB}} \simeq 0.04 \frac{\alpha+1}{b^{6.2}} \mathcal{R}_{\mathrm{to}}
$$

In steady state (justified given the short lifetimes $t_{\mathrm{UCXB}} \ll \tau$ of the bright UCXB phase) we can then estimate the number of UCXBs per $100 \mathrm{NSs}$ at present in a cluster as

$$
N_{100} \simeq 100 \mathcal{R}_{\mathrm{UCXB}} t_{\mathrm{UCXB}} .
$$

The lifetime $t_{\mathrm{UCXB}}$ depends on the minimum luminosity for a system to be classified as an UCXB. For our estimates we adopt a minimum luminosity comparable with the observed minimum in our Galaxy, $L_{\mathrm{x}} \simeq 10^{36} \mathrm{erg} \mathrm{s}^{-1}$. The corresponding lifetime is $t_{\mathrm{UCXB}} \simeq 10^{8} \mathrm{yr}$ (e.g., Rasio et al. 2000).

The present mass of a cluster $M_{\mathrm{t}}$ is always less than its initial mass $M_{\mathrm{t} ; 0}=f_{\mathrm{ML}}^{-1} M_{\mathrm{t}}$, where $f_{\mathrm{ML}}$ is the total mass loss fraction. About $40 \%$ of the initial mass is lost just through stellar winds and $\mathrm{SN}$ explosions, so that $f_{\mathrm{ML}}<0.6$. Without tidal mass loss (Joshi et al. 2001), and adopting a lower mass cutoff of $0.1 M_{\odot}$ in the IMF of Kroupa (2002), we expect about $1 \mathrm{NS}$ per $65 M_{\odot}$ of mass at present (see also Ivanova et al. 2004a). About 5\% of these NSs will be retained, depending on the escape velocity and the NS natal kick velocity distribution (Ivanova et al. 2004a). The corresponding minimum number of UCXBs expected (without any tidal mass loss) is then

$$
N_{\min } \sim 8 \times 10^{-4} M_{\mathrm{t}} \mathcal{R}_{\mathrm{UCXB}} t_{\mathrm{UCXB}},
$$

where $M_{\mathrm{t}}$ is in $M_{\odot}$.

In Table 1, we show numerical results for several Galactic clusters: all clusters where a UCXB has been identified, and 47 Tuc (which does not contain any bright LMXB).
The probability of finding a bright UCXB in a cluster like 47 Tuc is only about 23\%. NGC 6652 has poorly measured parameters (see Note for Table 1), and our numbers for this cluster are necessarily uncertain. Two clusters, NGC 6624 and NGC 6712, are thought to have very eccentric orbits and to be on the verge of complete disruption in the Galactic tidal field (Richtler et al. 1994; Gnedin et al. 1999; Andreuzzi et al. 2001). This suggests that they may have had much higher mass and density in the past. Indeed, observations show that NGC 6712 has a strikingly unusual mass function for stars below the turn-off (Andreuzzi et al. 2001) and this can only be explained if the cluster has lost more than 99\% of its initial mass (Takahashi \& Portegies Zwart 2000) ${ }^{4}$.

\section{DISCUSSION}

For Galactic clusters, our estimates indicate that it is quite possible for all observed UCXBs to have been formed through RG-NS collisions (Table 1). For extragalactic clusters, we can crudely estimate the expected total number of UCXBs in a galaxy by integrating over the cluster mass function and assuming some average formation rate per NS in all clusters. As an example, consider the case of M87 (Jordán et al. 2004). We adopt a power-law cluster IMF with $\alpha=-2$ (Kravtsov \& Gnedin 2004). With an average formation rate $\mathcal{R}_{\mathrm{UCXB}} \sim 2 \times 10^{-12}-4 \times 10^{-11} \mathrm{yr}^{-1}$ per NS (assuming that M87 clusters have structural parameters distributed roughly like those of Galactic clusters) and $t_{\mathrm{UCXB}} \sim 10^{6} \mathrm{yr}$ (corresponding to $L_{x}>10^{37} \mathrm{erg} \mathrm{s}^{-1}$, near the detectability limit for M87), we find that $\sim 10-100$ UCXBs are expected in the 1688 identified clusters, in rough agreement with the 58 detected LMXBs associated with these clusters.

In several galaxies, the probability of finding a bright LMXB in a cluster appears to correlate strongly with cluster metallicity (Kundu et al. 2002, 2003; Jordán et al. 2004). As there is no strong dependence of the RG-NS collision rate on metallicity, in our scenario, we have to interpret this trend as due to other factors, such as the metallicity dependence of the IMF, of the number of NSs formed in the cluster, or of the NS retention fraction. In other words, the metallicity dependence must appear through the number of NSs rather than through $\mathcal{R}_{\mathrm{UCXB}}$. This is difficult to verify, since there are no well established theoretical or observational predictions on how the IMF and NS natal kicks change with metallicity. A1ternatively, a strong metallicity dependence of $t_{\mathrm{UCXB}}$ is also possible, with higher metallicity systems having longer lifetimes as bright sources (cf. Maccarone et al. 2004).

Our conclusions are fairly robust, independent of assumptions and in spite of some large theoretical uncertainties. We now examine a few of the most important ones. Binaries were neglected in our analysis. This implies that our estimated collision rate is a lower limit, as interactions involving binaries always increase this rate (Fregeau et al. 2004). However, the effects of binaries on collision rates in very dense clusters today are likely to be small because core binary fractions in these clusters are very small, typically a few percent at most. This is known observationally (Cool \& Bolton 2002) and expected theoretically (Fregeau et al. 2003; Ivanova et al. 2004b). Another important assumption we made is that postcollision binaries do not circularize. As seen in Fig. 1, high

4 After significant mass loss, and depending on its initial density profile, the cluster could undergo strong gravothermal oscillations (Takahashi \& Portegies Zwart 2000), so that a UCXB could also have formed when the core had a much higher density during a recent, brief episode of core collapse. 
eccentricities are an important factor in keeping merger times short. However, one can also see directly from Fig. 1 that, even if all binaries were able to circularize quickly (compared to the GR merger time), a large fraction of post-collision systems would still merge in less than the cluster age. Based on the results of Sec. 2 and the relation between post-collision semimajor axis and collision parameters derived from our SPH simulations, we estimate this fraction to be about $70 \%$. Thus, even under the extreme assumption that all systems circularize, the rate of UCXB formation would still be within a factor of 2 of the total RG-NS collision rate. One possible further complication could come from the residual gas left bound to the RG core. All our collision calculations suggest that the mass left bound to the $\mathrm{RG}$ core is $\sim 0.1 M_{\odot}$. Although there are many theoretical uncertainties, it is possible that this is sufficient to reconstitute a RG envelope (Castellani et al. 1994). In this case, the orbit would likely circularize, and stable MT from the reconstituted RG onto the NS would occur. However, the Roche lobe in the post-collision binary is smaller than the equilibrium radius of the $R G$, so that the MT proceeds on a thermal timescale and the corresponding bright LMXB phase lasts only $\sim 10^{5} \mathrm{yr}$, making detection unlikely. In addition, the total mass accreted by the NS will be only $\sim 10^{-3} M_{\odot}$, which is not sufficient to produce a recycled millisecond pulsar.

\section{ACKNOWLEDGMENTS}

We thank R. Bi, S. Fleming, V. Kalogera, M. Rosenfeld and B. Willems for helpful discussions. This work was supported by NSF Grants AST-0206276 and AST-0353997, NASA Grants NAG5-12044 and NNG04G176G, and a Chandra Theory grant.

\section{REFERENCES}

Andreuzzi, G., De Marchi, G., Ferraro, F. R., Paresce, F., Pulone, L., \& Buonanno, R. 2001, A\&A, 372, 851

Bildsten, L., \& Deloye, C.J. 2004, ApJ, 607, L119

Castellani, V., Luridiana, V., \& Romaniello, M. 1994, ApJ, 428, 633

Chakrabarty, D. 2004, to appear in Binary Radio Pulsars, ASP Conf. Ser., ed. F.A. Rasio \& I.H. Stairs astro-ph/0408004

Clark, G.W. 1975, ApJ, 199, L143

Cool, A.M., \& Bolton, A.S. 2002, ASP Conf. Series, Vol. 263: Stellar Collisions, Mergers and their Consequences, 163

Davies, M.B., Benz, W., \& Hills, J.G. 1992, ApJ, 401, 246

Deloye, C.J., \& Bildsten, L. 2003 ApJ, 598, 1217

Djorgovski, S. 1993, ASP Conf. Ser. 50: Structure and Dynamics of Globular Clusters, 373

Deutsch, E.W., Margon, B., \& Anderson, S.F. 2000, ApJ, 530, L21

Fregeau, J.M., Cheung, P., Portegies Zwart, S.F., \& Rasio, F.A. 2004, MNRAS, 352, 1

Fregeau, J.M., Gürkan, M.A., Joshi, K.J., \& Rasio, F.A. 2003, ApJ, 593, 772

Gnedin, O.Y., Zhao, H., Pringle, J.E., Fall, S.M., Livio, M., \& Meylan, G. 2002, ApJ, 568, L23 (supplementary online data at wwwint.stsci.edu/ ognedin/gc/vesc.dat)

Gnedin, O.Y., Hernquist, L., \& Ostriker, J.P. 1999, ApJ, 514, 109

Grebenev, S.A., et al. 2004, ATEL 350

Hurley, J.R., Pols, O.R., \& Tout, C.A. 2000, MNRAS, 315, 543

Ivanova, N., Belczynski, K., Kalogera, V., Rasio, F.A., \& Taam, R.E. 2003, ApJ, 592, 475

Ivanova, N., Fregeau, J.M., \& Rasio, F.A. 2004a, to appear in Binary Radio Pulsars, ASP Conf. Series, ed. F.A. Rasio \& I.H. Stairs ‘astro-ph/0405382
Ivanova, N., Belczynski, K., Fregeau, J.M., \& Rasio, F.A. 2004b, MNRAS, in press

Jordán, A., et al. 2004, ApJ, 613, 279

Joshi, K.J., Nave, C.P., \& Rasio, F.A. 2001, ApJ, 550, 691

Kalogera, V., \& Webbink, R.F. 1996, ApJ, 458, 301

Kalogera, V., Henninger, M., Ivanova, N., \& King, A.R. 2004, ApJ, 603, L41 Kroupa, P. 2002, Science, 295, 82

Kravtsov, A.V., \& Gnedin, O.Y. 2004, ApJ, in press

Kundu, A., Maccarone, T. J., \& Zepf, S. E. 2002, ApJ, 574, L5

Kundu, A., Maccarone, T. J., Zepf, S. E., \& Puzia, T. H. 2003, ApJ, 589, L81

Lombardi, J.C., Dooley, K., Proulx, Z., Ivanova, N., Rasio, F. 2004, in prep.

Maccarone, T.J., Kundu, A., \& Zepf, S.E. 2004, ApJ, 606, 430

Peters, P.C. 1964, Phys. Rev., 136, 1224

Podsiadlowski, P., Rappaport, S., \& Pfahl, E.D. 2002, ApJ, 565, 1107

Pryor, C. \& Meylan, G. 1993, ASP Conf. Ser. 50: Structure and Dynamics of Globular Clusters, 357

Pylyser, E., \& Savonije, G.J. 1988, A\&A, 191, 57

Rasio, F.A., Pfahl, E.D., \& Rappaport, S. 2000, ApJ, 532, L47

Rasio, F.A., \& Shapiro, S.L. 1991, ApJ, 377, 559

Richer, H.B., et al. 2004, AJ, 127, 2771

Richtler, T., Grebel, E.K., \& Seggewiss, W. 1994, A\&A, 290, 412

Takahashi, K. \& Portegies Zwart, S. F. 2000, ApJ, 535, 759

van der Sluys, M. V., Verbunt, F., \& Pols, O. R. 2005, A\&A, 431, 647

Verbunt, F. 1987, ApJ, 312,L23

Webbink, R. F. 1985, IAU Symp. 113: Dynamics of Star Clusters, 113, 541

Note added in proof. - Bildsten \& Deloye (2004) pointed out that the observed break around $5 \times 10^{38} \mathrm{erg} \mathrm{s}^{-1}$ in the X-ray luminosity functions of elliptical galaxies could be explained naturally if all donors in UCXBs had initial masses clustered near $\sim 0.1 M_{\odot}$. This is precisely what is predicted by our scenario. 